\title{
Dermatoglyphic Studies among the Dumagat-Remontado Tribal Population of the Philippines
}

\author{
Sally B. Gutierez, ${ }^{1,2}$ John Lou S. Lucenario, ${ }^{1,3}$ and Mark Jason T. Yebes ${ }^{3}$ \\ ${ }^{1}$ Division of Curriculum and Instruction, College of Education, University of the Philippines, Diliman, 1101 Quezon City, Philippines \\ ${ }^{2}$ Elementary School Science Group, National Institute for Science and Mathematics Education Development, \\ University of the Philippines, 1101 Quezon City, Philippines \\ ${ }^{3}$ San Jose National High School, Rodriguez, 1860 Rizal, Philippines \\ Correspondence should be addressed to Sally B. Gutierez, sallybgutierez@yahoo.com \\ and John Lou Lucenario, johnloulucenario@gmail.com
}

Received 13 September 2012; Revised 22 November 2012; Accepted 26 November 2012

Academic Editor: Kaushik Bose

Copyright (C) 2012 Sally B. Gutierez et al. This is an open access article distributed under the Creative Commons Attribution License, which permits unrestricted use, distribution, and reproduction in any medium, provided the original work is properly cited.

\begin{abstract}
Dermatoglyphics is the study of dermal ridges. Since dermatoglyphic characters are recognized as a unique individual trait and follow a multifactorial or polygenic pattern of inheritance, it is necessary to understand the variations leading to this pattern. The present paper, through appropriate descriptive approach, has revealed the variation in the pattern of inheritance in the TFRCs of the Dumagat-Remontados from Puray, Rodriguez, Rizal, Philippines. The study also reported a large discrepancy on the total fingerprint ridge count among the Dumagat-Remontados compared to established researches. Also, the increased heterozygosity due to genetic admixture led to the dominant occurrence of the loop pattern (ulnar) in the fingerprints of this group of indigenous people as compared to few established data stating that the frequency of whorl patterns dominates the universal population and other Asian racial groups. In this study, a special feature on the fingerprint patterns of the pure Dumagat-Remontados was reported. Aside from the high frequency of loops and whorls, a distinct club dent, concluded to be a unique feature found among this tribal population, was noted from at least one of the fingers of the majority of the pure sample.
\end{abstract}

\section{Introduction}

Dermatoglyphics is a field that has attracted a great number of scientists from across genetics, medicine, and anthropology to explore links on genetically determined diseases. Moreover, for many years, fingerprints were used as a means of identification and authentication based on their individual peculiarities and uniqueness. According to Ismail et al. [1], the fingerprint patterns are almost certainly influenced by the interaction of several genes; thus, the ridge patterns are distinct and unique for every individual making it widely used as one of the tools in criminal identification worldwide. They are individual-specific and are highly heritable [2], and as such, they are noted to play a major role in medical and genetic studies of human biology research as well as population studies. In the medical field, these traits are used in the diagnosis of chromosomal abnormalities [3], while in genetic studies they are used as an easily applied and inexpensive tool for estimating the genetic distances between populations [2]. In a study by Eboh and Arko-Boham [4], more than $60 \%$ of all fingerprints have the same basic pattern but no two fingerprints are exactly the same.

Dermatoglyphic study is comprised of two main aspects: patterns and total fingerprint ridge counts (TFRCs). Patterns are primarily due to the convoluted layers of cells of the epidermis wherein the peaks of the ridges are the locations of the pores of the sweat glands. Figure 1 shows the structure of the fingerprint highlighting the delta or the triradius and the core which establishes the total fingerprint ridge count (TFRC). TFRC is the summation of the ridge counts from the fingers of both hands.

Fogle [5] mentioned in his study that fingerprints are formed during embryonic development and the patterns remain unchanged during postnatal development. However, 


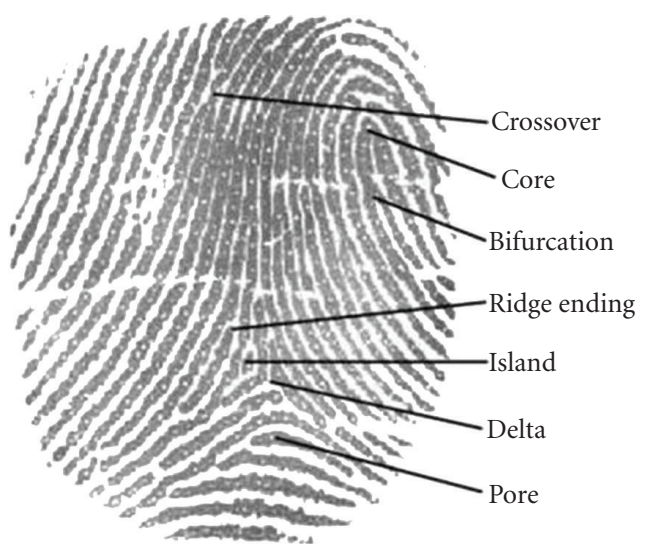

FIGURE 1: Structure of a fingerprint. http://perso.orange.fr/fingerchip/biometrics/types/fingerprint.htm.

extensive physical labor can wear down ridges and that scars can distort the patterns. Ridge depth is influenced by age such that both young children and older adults have thinner ridges.

Human fingerprint is characterized by various types of ridge patterns classified as an arch, a loop, or a whorleach has a unique characteristic with respect to a reference point called a triradius. The arch is the least frequent pattern and is subdivided into either plain or tented arch consisting of at least one upthrusting ridge, which tends to bisect superior ridges at right angles. The loop pattern is more complicated classified as either ulnar (with a triradius that is on the side of the thumb, with the loop opening towards the pinky finger) or radial (with a triradius that is on the side of the pinky finger, with the loop opening towards the thumb). The whorl has two triradii and has four types: plain whorl, central pocket whorl, double-loop whorl, and the superwhorl. According to Holt [6], a ridge count is the number of ridges intervening between the triradius and the core or center which cuts or touches a straight line joining these two points in a finger. Each of the patterns has a unique method of obtaining the TRCs such that arch patterns have zero ridge count, loop pattern ridge count is obtained by counting the number of ridges between the triradius and the center or core of the pattern, and whorl pattern ridge count is made from each triradius to the center of the fingerprint and the higher of the two possible counts is recorded. Summing all the ridge counts for each of the ten fingers makes up the total fingerprint ridge counts.

Ridge count is related to two different timed events: the timing of the onset of volar pad regression versus the timing of the onset of primary ridge formation [7]. For example, if ridges did not start to form until the pad was in a more regressed state, less ridges would form, which would lower the ridge count [7]. However, if there was late regression of the volar pad, it would result in more ridges forming on the pad, which would raise the ridge count [7]. A person's fingerprint is permanent even before they are born. It is the representation of the dermal ridges in each fingertip [8]. Around 6-8 weeks of conception, the volar pads (ball-like structures that make up the contour of the fetal hand) form and begin to recede at around 10-12 weeks. While the volar pads recede on the 13th week, dermal ridges take place, become visible, and take the shape of the receding volar pads until the 21st week to which the formations of the fingerprint are complete [9]. While this may break down into a relatively simple process, there are many components and anatomical changes that go into making a fingerprint a permanent and unique structure that is seen on the fingers. Cummins [10] found that the configuration of ridge patterns is determined partly by heredity and partly by accidental or environmental influence, which produces stress and tension in their growth during fetal life. Environmental factors include diet and chemical intake of the mother, hormone levels, and radiation levels which can directly or indirectly affect the ridge count of the developing fingerprints [7].

Dermatoglyphics, as termed by Cummins [10], is the study of the dermal ridge counts and patterns in fingers, palms, and soles. Like any other phenotypic trait, fingerprints and toeprints may also be controlled by genes. It is considered a classical model of polygenic inheritance (several genes are involved in the inheritance) [11] as well as a multifactorial trait (ridges can be counted as total ridge count) [12]. According to Penrose [12], seven genes are thought to be involved in the fingerprint formation. In polygenic inheritance, the genes that confer this follow Mendel's laws, but, together, they do not produce a single-gene phenotypic ratio. Instead, they all contribute to the phenotype without being dominant or recessive to each other. In today's forensic studies, human fingerprints have been used extensively to establish one's biometric identity especially in workplaces and other legal matters. For the case of the Dumagat-Remontados, this would be helpful such that this heritable trait may set another step to establish their uniqueness as a distinct group of indigenous people in the Philippines.

The Dumagat-Remontados, identified as a Negrito ethnolinguistic group, derive their name from the Spanish verb "remontar," meaning, "to flee to the hills," "to frighten away," or "go back to the mountains." These indigenous peoples (IPs) are said to be the descendants of lowlanders who opted to live in the mountains to avoid subjugation by the Spaniards. Subsequently, they intermarried with the Negrito groups. The Remontados are also referred to as Dumagat-Remontados and they prefer to be called "taga-bundok" (from the mountains) or "magkakaingin" (those who practice kaingin).

The Remontados are mixed-blooded (genetic admixture) group of people; hence, they have a combination of a Negrito and a lowland Filipino features with deep brown skin, average height of 128 centimeters, curly-to-straight hair, high cheekbones, small slightly flared noses, Mongol-type eyes, and teeth that are sometimes filed and red from chewing betel nut. They also have lean and small built but with strong firm muscles and well-developed extremities including broad feet, because of physical daily work in the field such as climbing hills and hiking in the forests. In the Philippines, although the Dumagat-Remontados are seminomadic, there is no reported case of heavy out-migration. They roam in a specific geographic range which they consider their home base. 
In 1936, the total population of Remontados in the Province of Eastern Rizal, Philippines, was around 2,650. After 45 years, this was recorded to around 2,750. The group's population growth is almost stationary as compared to the province's non-Remontado populace that has increased five times in the same time period.

In most dermatoglyphic studies conducted, fingerprint ridge counts were given emphasis highly by many researchers in terms of their quantitative aspect. In the study conducted by Holt [11], ridge counts fit the multifactorial model extremely well, although Spencer and colleagues [13] discovered a direct evidence for substantial major-gene effect especially on the inheritance of arches. From their study, the frequency inheritance of arches in different racial groups may range from 0.02 in Oriental populations [14] to 0.16 in Pygmies [15], although, in general, most arches are inherited with a frequency ranging from 0.03 to 0.06 . In addition to these reported data on major-gene effect, there was an examination of the "trait arch on any digit" and its possible linkage to several blood and serum group markers (with the haptoglobin locus) confirmed that this trait exhibits almost complete inheritance [16].

Fingerprint inheritance was also investigated in terms of the general distribution of the patterns in relation to the different blood groups [17]. In the study of Bharadwaja et al. [17], it was found out that loops were more frequent $(51.87 \%)$ which was followed by the whorls $(35.83 \%)$ and the arches are the least frequent $(12.30 \%)$. In this study, it was also noted that there is a significant relationship among the fingerprint patterns, the Total Ridge Counts and the different blood groups such that loops were frequently found in blood group A and whorls and higher total fingerprint ridge counts in the blood group B. In contrast to this study, it was reported that in Ghanian population, the distribution of the fingerprint patterns does not follow any fixed or regular trend; therefore, it cannot be used to identify siblings and blood relations even in twins [4]. However, it can still be used to identify individuals with the minutiae (Galton's details) as the distinguishing feature. The minutiae is a term coined by Galton to refer to the small peculiarities present along the length of every isolated ridge, or characteristic points which has inter- and intrapopulation variability.

Another study reported that the gross distortions in the patterns can occur in association with any limb malformation of early prenatal onset [3]. Unusual dermatoglyphic patterns were mostly found in individuals with Down syndrome [18], in trisomic individuals [19], and in individuals with congenital cutis laxa [3]. This is, however, not surprising since abnormal karyotypes usually lead to multiple morphological abnormalities.

In most of the previous studies, there was really an emphasis on fingerprints because of their uniqueness to every individual. In this paper, an investigation of the pattern of fingerprints in Dumagat-Remontados was done which aims to investigate whether the TFRCs of this Philippine tribal population follow the polygenic pattern of inheritance. Comparisons of the TFRCs within gender (males and females), genotypic lineage (pure and hybrid), and the frequencies of occurrences of the common fingerprint pattern types were also examined.

\section{Methods}

Dumagat-Remontados from Puray, Rodriguez, Province of Rizal, Philippines, were chosen purposively as respondents of this study. The total fingerprint ridge counts (TFRCs) and the fingerprint patterns of fifty (50) Dumagat-Remontados were recorded by manual counting and identification using a magnifying lens. TFRC was obtained by summing the ridge counts from all fingers of both hands. Primary patterns were observed and TFRCs were counted as follows: ridge count in an arch is counted as zero, in a loop, the number of ridges between the center and the triradius was counted, in a whorl, the numbers of both the radial and ulnar triradius were counted and the greater of the two was used. Prior to ridge counting and pattern identification, the respondents were interviewed of their genotypic lineages (e.g., pure genotypic lineages are those whose parents are both DumagatRemontados and hybrid genotypic lineages are those with either a Dumagat-Remontado father or mother).

All data were tabulated and statistically treated. Descriptive statistics were used to establish significant frequencies in the sample. Chi-square test of significance was also computed to determine whether there are significant differences in the occurrences of patterns in every individual. Comparison of the mean TFRCs for gender and between pure and hybrid individuals in the sample were obtained separately. Hybrid individuals in the sample, however, were limited up to the first filial generation. It is important to note here that the genetic admixture of the tribal population was due to the intermarriage of the tribal members with lowland Filipinos. It can also be inferred that the stability of the population number of this tribal group was due to the hybridization (intermarriage) of the pure Dumagat-Remontado with individuals outside their tribe.

\section{Results and Discussions}

Results revealed that in this indigenous group of people, the attempt to uncover the mode of inheritance of dermatoglyphic traits is conclusive and that it fits the multifactorial model or polygenic pattern of inheritance. Total fingerprint ridge counts range from 91 to 279 with a mean of 203.12, standard deviation of 37.57 with a slight negatively-skewed distribution value of -0.877 (Figure 2 ).

Table 1 shows that males have higher TFRCs $(\mu=$ $217.5 ; \mathrm{SD}=33.79)$ than females $(\mu=195.03 ; \mathrm{SD}=37.66)$. The presence of arches in any individual greatly reduces the value of a person's TFRC [16]. From the data, it was found out that the occurrence of arches is less frequent which accounts to the significant increase of the TFRCs in the sample.

Holt [11] said that the size of the volar pad played a dominant role in determining the TRCs such that larger volar pads take longer recession thereby giving the primary ridge formation a longer time to form ridges resulting in a higher ridge count. This evidence supports the high TFRCs 


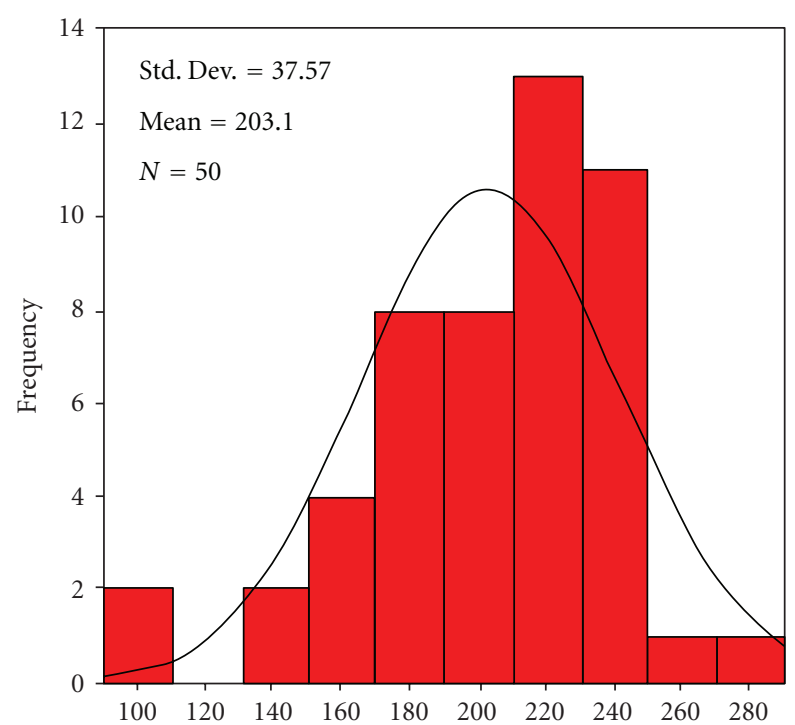

Figure 2: Total fingerprint ridge count distribution among Dumagat-Remontados.

TABLE 1: Descriptive statistics of the TFRC of males and females.

\begin{tabular}{lcc}
\hline \multirow{2}{*}{ Descriptive Statistics } & \multicolumn{2}{c}{ Fingerprints } \\
& Male $(N=18)$ & Female $(N=32)$ \\
\hline Mean & 217.5 & 195.03 \\
Std. deviation & 33.79 & 37.66 \\
Range & 127 & 157 \\
Minimum & 152 & 91 \\
Maximum & 279 & 248 \\
\hline
\end{tabular}

TABLE 2: Frequencies of each fingerprint pattern type.

\begin{tabular}{lcc}
\hline Pattern types & Frequency & Percentage \\
\hline Whorl & 212 & 42.4 \\
Ulnar loop & 271 & 54.2 \\
Radial loop & 6 & 1.2 \\
Arch & 11 & 2.2 \\
\hline Total & 500 & 100.0 \\
\hline
\end{tabular}

of the population since most of these indigenous people have wide volar pads as shown in Figures 3(a) and 3(b).

Table 2 and Figure 4 reveal that from among the Dumagat-Remontados, the occurrence of ulnar loop patterns is evident $(54.2 \%)$ followed by the whorl pattern (42.4\%). These data are supported by previous studies stating that the average frequency of whorl, ulnar loop, radial loop, and arch in whole-world population is $70 \%$, $<25 \%,<1 \%$, and $5 \%$, respectively $[15,20]$. In addition, the frequency of arches in various racial groups has been reported to range from 0.02 in Oriental populations [14] to as high as 0.16 [15] in Pygmies, although most range from 0.03 to 0.06 . Particularly, studies on Asian racial groups suggest that in Mongoloid population from East Asia, the frequency of whorl patterns is high (about $46 \%$ ) and the frequency of arches is quite low [21], which was also observed

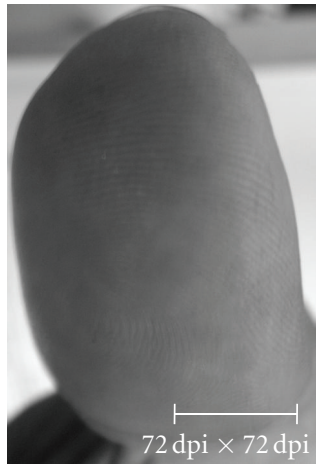

(a)

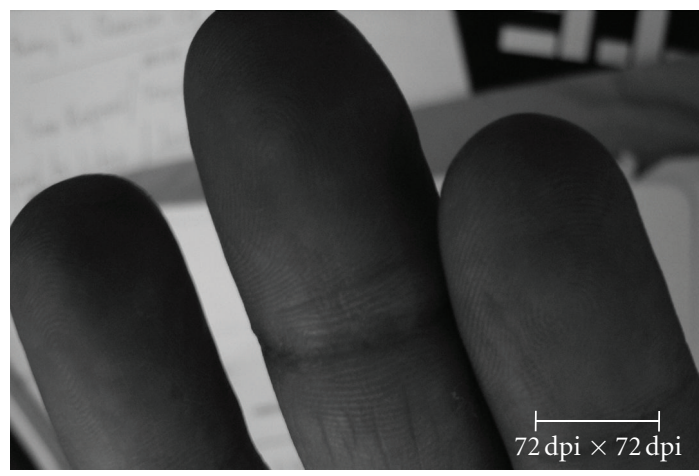

(b)

Figure 3: A typical volar pad of a Dumagat-Remontado.

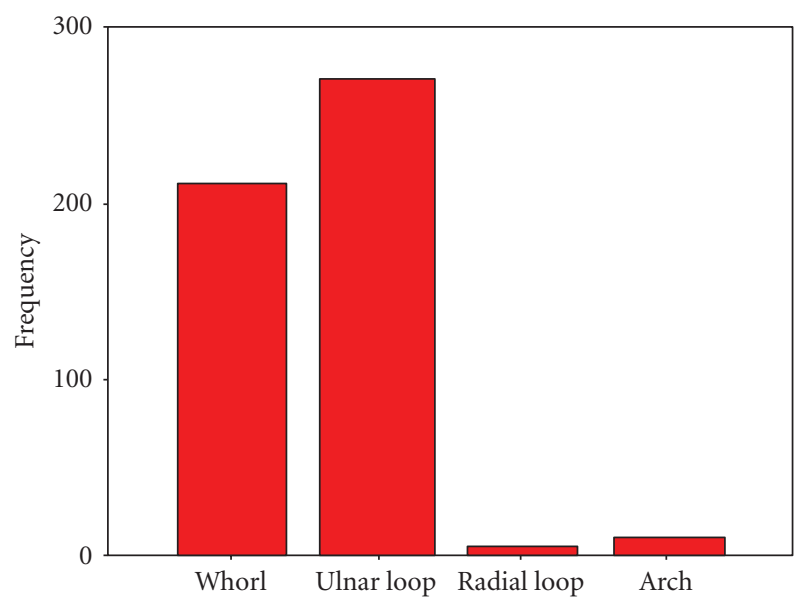

FIGURE 4: Frequency distribution of the different fingerprint pattern types.

in this study. Hence, these figures give evidence of a universal trait rather than a rare syndrome confined to a specific population.

The difference in the frequencies of occurrences of the fingerprint patterns within gender (male and female) and genotypic lineage (pure and hybrid) was also tested using the chi-square test of significance and results show stable significant differences $(P>\alpha=0.01)$ (Table 3). This contrasts previous study of Acree [22] that females have higher 


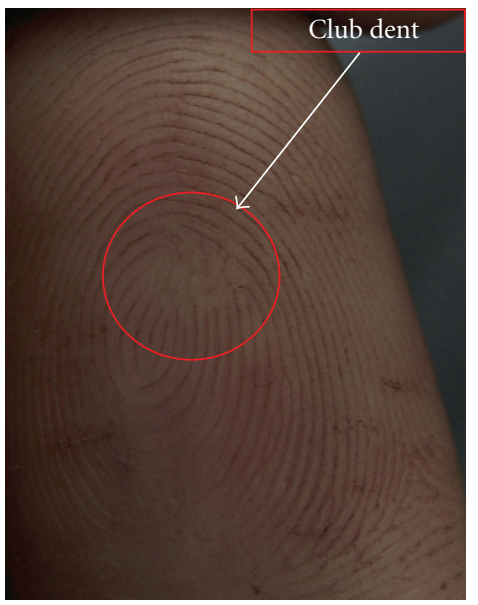

Figure 5: Observed unique feature from the fingerprints of the Dumagat-Remontado.

TABle 3: Chi-square values for frequency distributions of the different fingerprint pattern types in selected relationships.

\begin{tabular}{lcc}
\hline Relationships & Finger prints & $P$ value \\
\hline Male-female & 16.909 & 0.001 \\
Pure-hybrid & 18.39 & 0.000 \\
\hline
\end{tabular}

Critical value at $\alpha=0.01 ; d f=3$.

ridge density since they have "finer" epidermal ridge details compared to males.

In this study, a special feature on the fingerprint patterns of the pure Dumagat-Remontados was observed. Moreover, the high frequencies of loop occurrence might have been due to the genetic admixture that happened in the current population. Dermatoglyphic traits are steadily inherited and show slow changes within a population, but because of genetic admixture, the variability might be highly evident [23]. The fact that this trait is said to be influenced by many genes, the effect of genetic admixture may increase the prevalence of gene heterozygosity. This principle was evident in this study such that due to intermarriage, increased heterozygosity in the current population led to high frequency of occurrence of loop patterns. This supports the study of Cheng and colleagues [23] that in the admixed population of Chinese, the increase of simple whorl frequency may be just a ripple effect of the decrease of simple arch frequency. Aside from the high frequency of occurrence of loops and whorls, a distinct club dent was noted from atleast one of the fingers from majority of the pure individuals in the sample as shown in Figure 5. Initially, this was noted to be a worn fingerprint structure due to extensive work, but the observation from around $40 \%$ of the pure individuals in the sample led to a conclusive statement that this might be a unique feature among the Dumagat-Remontado tribal population in the Philippines.

\section{References}

[1] E. Ismail, S. A. Razak, L. Selamat et al., "Dermatoglyphics: comparison between Negritos Orang Asii and the malays,
Chinese and Indian," Sains Malaysiana, vol. 38, no. 6, pp. 947952, 2009.

[2] G. Temaj, J. Milicic, T. K. Juric et al., "Comparative analysis of dermatoglyphic traits in Albanian and Turkish population living in Kosovo," Collegium Anthropologicum, vol. 33, no. 4, pp. 1001-1005, 2009.

[3] H. Pour-Jafari, A. Sarihi, M. Hashemzadeh, and D. D. Farhud, "Dermatoglyphic observations in an Iranian girl affected with congenital cutis laxa (autosomal recessive)," Iranian Journal of Public Health, vol. 32, no. 2, pp. 12-15, 2003.

[4] D. Eboh and B. Arko-Boham, "Genetics and fingerprints: hereditary identity in Ghanaian individuals," Nigerian Annals of Natural Sciences, vol. 8, no. 1, pp. 19-24, 2008.

[5] T. Fogle, "Using dermatoglyphics from Down syndrome and class populations to study the genetics of a complex trait," (Dermatoglyphics 99-121), Association for Biology Laboratory Education (ABLE), http://www.zoo.utoronto.ca/able.

[6] S. B. Holt, "Epidermal ridge-counts," Collegium Anthropologicum, vol. 1, no. 3, pp. 97-106, 1979.

[7] K. Wertheim and A. Maceo, "The critical stage of friction ridge and pattern formation," Journal of Forensic Identification, vol. 52, no. 1, pp. 35-85, 2002.

[8] H. S. Kahn, R. Ravindranath, R. Valdez, and K. M. V. Narayan, "Fingerprint ridge-count difference between adjacent fingertips (dR45) predicts upper-body tissue distribution: evidence for early gestational programming," American Journal of Epidemiology, vol. 153, no. 4, pp. 338-344, 2001.

[9] W. J. Babler, "Prenatal selection and dermatoglyphic patterns," American Journal of Physical Anthropology, vol. 48, no. 1, pp. 21-27, 1978.

[10] H. Cummins, "Palmar and plantar epidermal ridge configuration (Dermatoglyphics) in Europeans and Americans," American Journal of Physical Anthropology, vol. 179, pp. 741-802, 1926.

[11] S. B. Holt, The Genetics of Dermal Ridges, C.C. Thomas, Springfield, Ill, USA, 1968.

[12] L. S. Penrose, "Dermatoglyphics," Scientific American, vol. 221, no. 6, pp. 72-83, 1969.

[13] M. A. Spencer, R. C. Elston, K. K. Namboordiki, and W. S. Politzer, "Evidence for a possible major gene in absolute finger ridge count," Human Heredity, vol. 23, no. 5, pp. 414-421, 1973.

[14] C. C. Plato, Variation and Distribution of the Dermatoglyphic Features in Different Populations, Penrose Memorial Colloquium, Berlin, Germany, 1973.

[15] H. Cummins and C. Mildo, Fingerprints, Palms and Soles, Dover, New York, NY, USA, 1961.

[16] M. W. Anderson, B. Bonne-Tamir, D. Carmelli, and E. A. Thompson, "Linkage analysis and the inheritance of arches in a Habbanite isolate," American Journal of Human Genetics, vol. 31, no. 5, pp. 620-629, 1979.

[17] A. Bharadwaja, P. K. Saraswat, S. K. Agrawal, P. Banerji, and S. Bharadwaja, "Pattern of finger-prints in different ABO blood groups," Journal of Forensic Medicine and Toxicology, vol. 26, no. 1, 2004.

[18] S. B. Holt, "Dermatoglyphics in mongolism," Annals of the New York Academy of Sciences, vol. 171, pp. 602-616, 1970.

[19] L. J. Ross, "Dermatoglyphic observation in a patient with trisomy 18," Journal of Pediatrics, vol. 72, no. 6, pp. 862-863, 1968.

[20] H. Gray, Grays Anatomy: Skin and Its Appendages, Elsevier, Churchill Livingstone, New York, NY, USA, 40th edition, 2008.

[21] M. K. Bhasin, H. Walter, and H. Danker-Hopfe, People of India: An Investigation of Biological Variability in Ecological, 
Ethno-Economic and Linguistic Groups, Kamla-Raj Enterprises, New Delhi, India, 1994.

[22] M. A. Acree, "Is there a gender difference in fingerprint ridge density?” Forensic Science International, vol. 102, no. 1, pp. 3544, 1999.

[23] X. Cheng, H. Li, S. Gupta, S. Pan, J. Huo, and L. Jin, “Dermatoglyphic changes during the population admixture between Kam and Han Chinese," HOMO_Journal of Comparative Human Biology, vol. 60, no. 2, pp. 143-157, 2009. 


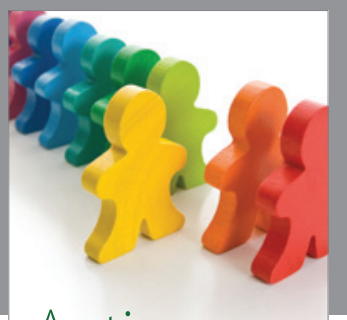

Autism

Research and Treatment
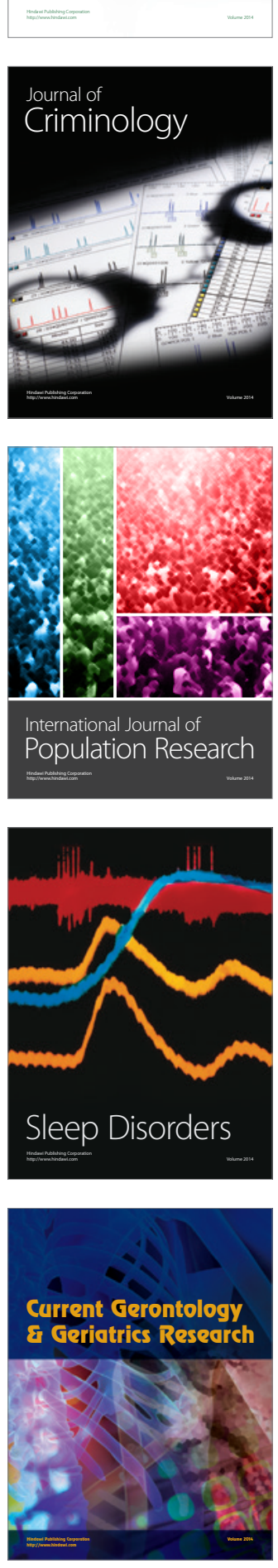
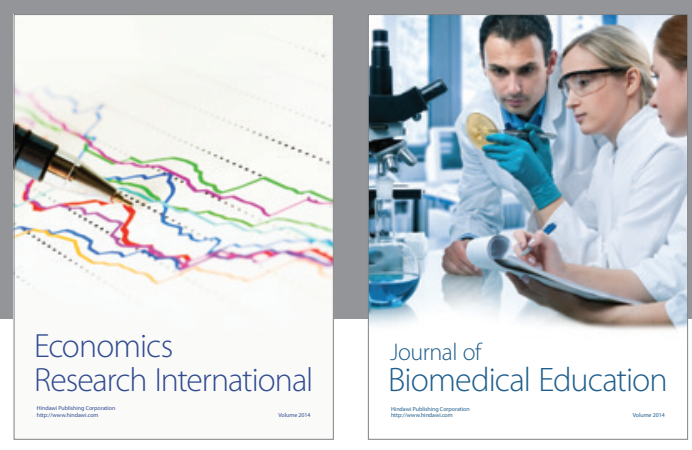

Journal of

Biomedical Education

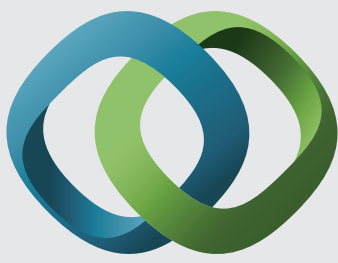

\section{Hindawi}

Submit your manuscripts at

http://www.hindawi.com
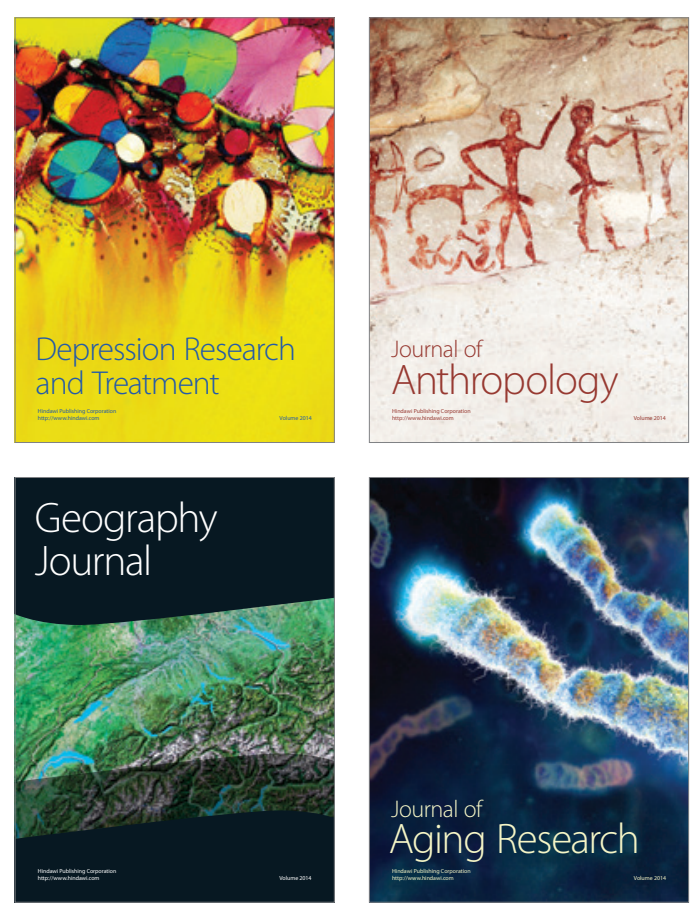

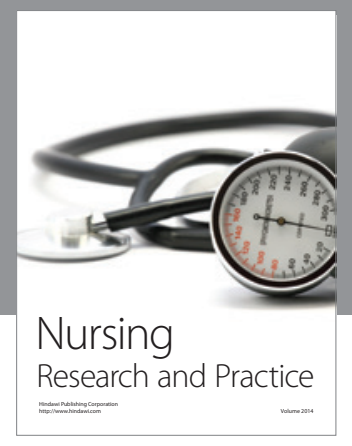

Nursing

Research and Practice

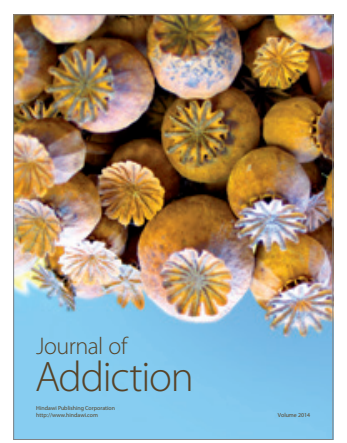

Child Development

Research

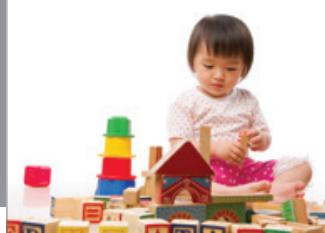

迥
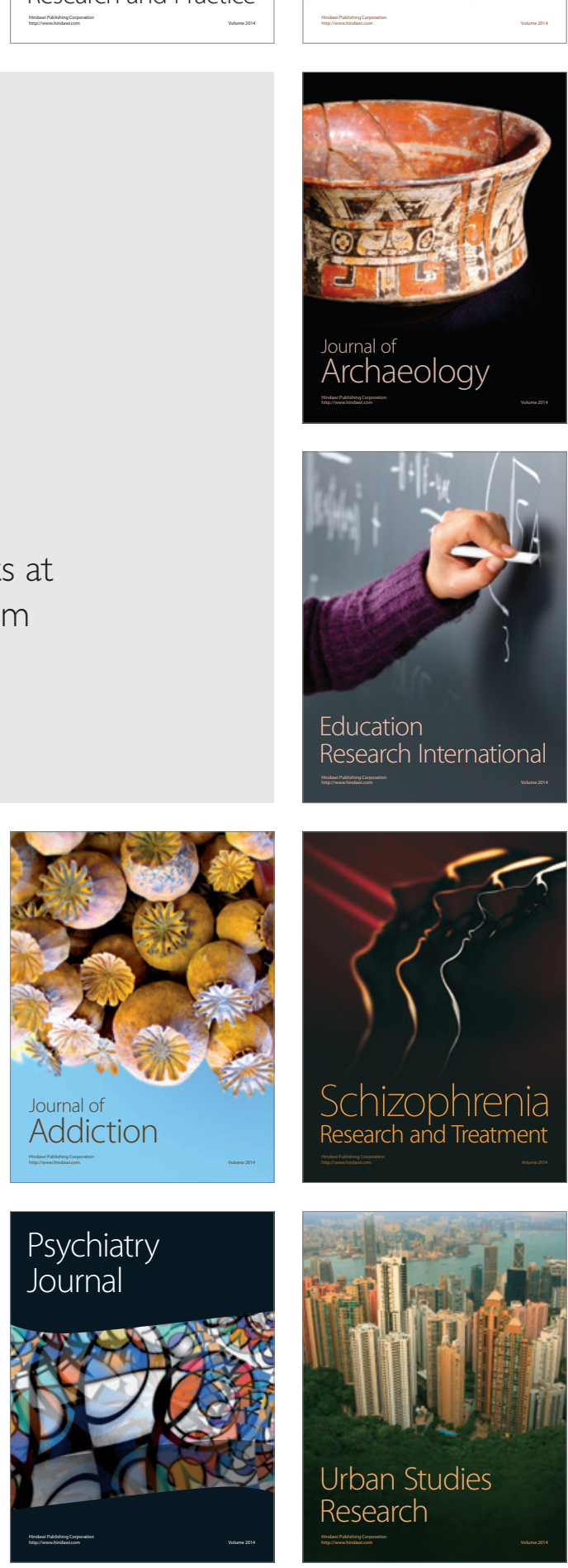